\title{
A new etiology for variant of Guillain-Barré syndrome: bariatric surgery
}

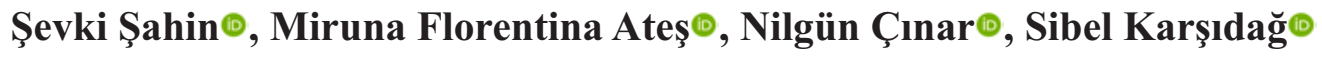 \\ Department of Neurology, Maltepe University School of Medicine, İstanbul, Turkey
}

DOI: $10.18621 /$ eurj.461760

\begin{abstract}
Bariatric surgery is an effective treatment for obesity. However, the number of acute or chronic neurological complications after bariatric surgery, including Guillain-Barré syndrome, is increasingly reported. We present here two cases which developed acute motor sensory polyneuropathy a couple months after bariatric surgery which rapidly progressed over the following month. Both patients used received parenteral vitamin B complex replacement after surgery. The first case responded well to intravenous immunoglobulin (IVIg) treatment. However, the second case required plasmapheresis and physical rehabilitation for recovery after IVIg treatment. It is thought that minerals, vitamins, and trace element deficiencies can develop after bariatric surgery. These deficiencies may trigger inflammatory and autoimmune mechanisms and cause acute polyneuropathies. In such cases, it should be kept in mind that immune therapies may be beneficial, as well as vitamins.
\end{abstract}

Keywords: Bariatric, complication, neuropathy, malnutrition, Guillain-Barré syndrome

Received: September 20, 2018; Accepted: December 11, 2018; Published Online: August 2, 2019

O besity is a growing problem and the necessity for bariatric intervention is apparent worldwide [1-3]. Neurological complications are increasingly recognized by the number of bariatric surgeries performed. These complications such as encephalopathy, optic neuropathy, myelopathy, radiculoplexopathy, and neuropathy can involve any level of the central and peripheral nervous system. Neuropathies after bariatric surgery have been reported in $5-16 \%$ of various studies [4-6]. Neurological complications may result from immune and inflammatory mechanisms, and some authors claim that the real underlying pathology originates from micro-nutritional deficiencies, such as a lack of vitamins $\mathrm{B}$ and $\mathrm{E}$ and trace elements $[5,6]$. Changes in incretins secreted from intestinal endocrine cells after gastrectomy are also blamed on a pathological mechanism $[7,8]$. In addition, there are very few studies reporting that polyneuropathy develops as a result of an autoimmune reaction after bariatric surgery $[9,10]$. Although the underlying mechanisms are still debated, there is, as yet, no general consensus for treatment [10-13]. We discuss the clinical findings and treatment response of two cases developing acute axonal sensory motor neuropathy (AMSAN) which as a variant of Guillain-Barré syndrome (GBS) related bariatric surgery.

\section{CASE PRESENTATION}

\section{Case 1}

A 27 -year-old female with $59.7 \mathrm{~kg} / \mathrm{m} 2$ body mass index (BMI) (height: $152 \mathrm{~cm}$, weight: $138 \mathrm{~kg}$ ), underwent a sleeve gastrectomy and was discharged

Address for correspondence: Miruna Florentina Ateş, MD., Assistant Professor, Maltepe University School of Medicine, Department of Neurology, Feyzullah Cad, No. 39, 3484 Maltepe, İstanbul, Turkey, E-mail: miruna.ates@gmail.com 
on a special diet including oral multivitamins and mineral and protein supplements. She had also been using intramuscular $1000 \mathrm{mcg}$ hydroxocobalamin, 100 $\mathrm{mg}$ thiamine and $100 \mathrm{mg}$ pyridoxine per week. Three months after surgery, she developed a burning sensation in her feet, and lower back pain. After this, weakness in both lower limbs increased progressively. Weight loss was almost $20 \mathrm{~kg}$ over this duration. Her examination showed normal mental function, intact cranial nerves, dystal muscle weakness (a grade of muscle strength of 4/5) with areflexia in lower extremities. Complete blood counts, vitamin B12, folate levels, and metabolic panels, electroneuromyography (ENMG) and magnetic resonanace imaging (MRI) of brain, cervical, thoracic and lumbar regions were found to be normal. Routine cerebrospinal fluid (CSF) analysis including protein, glucose, sodium and potassium levels were normal, in addition no inflammatory cell reaction was found. Her complaints got progressively worse. Over the following month she was unable to get up and walk without full physical assistance. Laboratory studies were repeated. Vasculitis panel, protein electrophoresis, and tumour markers were found negative. The new ENMG showed a severe, diffuse reduction in the amplitudes of the motor and sensory action potentials, particularly in the lower extremities. No conduction block or increase in distal latencies were noted. Needle examination showed fibrillation potentials and positive sharp waves in distal limb muscles. It was thought to be a case of AMSAN. Intravenous immunoglobulin (IVIg) treatment was started $0.4 \mathrm{~g} / \mathrm{kg} /$ day for 5 consecutive days and 2 $\mathrm{g} / \mathrm{kg} / \mathrm{month}$ for 6 consecutive months. Also, treatment with intramuscular B-complex vitamins were continued. She began walking short distances after six months. Weight loss was $45 \mathrm{~kg}$ over the 6 month period.

\section{Case 2}

A 19-year-old male with $50.5 \mathrm{~kg} / \mathrm{m} 2$ BMI (height: $1.78 \mathrm{~m}$, weight: $160 \mathrm{~kg}$ ) was submitted to a sleeve gastrectomy four months previous. A liquid high protein/low carbohydrate diet was instituted and intramuscular vitamine B complex per week was administered after surgery. He had numbness and pain in his legs and hands for 2 weeks. Muscle weakness in the lower extremities started in the feet and gradually ascended over the following days. A cranial nerve examination was normal. The predominant distal symmetric paresis was observed in the arm and leg muscles (grade of muscle strength of 3/5). Deep tendon reflexes were absent in the lower extremities. Routine blood chemistry analyses, including vitamin B12, folate levels, and vasculitis panel, were normal. An MRI of the brain, cervical and lumbar spine was normal. Routine CSF findings were also normal. ENMG showed a severe, diffuse reduction in the amplitudes of the motor and sensory action potentials, prolongation in distal latencies, and slowing of conduction velocities - particularly in the lower extremities. Needle examination showed acute denervation potentials in the muscles of the lower extremities. AMSAN has been established as a diagnosis. IVIg treatment was started at a dose of 0.4 $\mathrm{g} / \mathrm{kg} /$ day for 5 consecutive days. However, his muscle strength reduced to a grade of $1 / 5$ in the distal part of the extremities. Plasmapheresis was started 5 times daily on alternate days. In addition, treatment with intramuscular B-complex vitamins was maintained. Six weeks of physiotherapy was admistered after plasmapheresis. At his last examination his neurological condition was nearly normal. He had lost $90 \mathrm{~kg}$ over the 6 months.

\section{DISCUSSION}

Several bariatric procedures have been applied in the treatment of obesity, such as Roux-en-Y gastric bypass, adjustable gastric banding, and sleeve gastrectomy. Selection criteria for surgery includes $\mathrm{BMI} \geq 40 \mathrm{~kg} / \mathrm{m}^{2}$ without co-morbidities. If patients have diabetes mellitus, or obstructive sleep apnea, the $\mathrm{BMI}=35-40 \mathrm{~kg} / \mathrm{m}^{2}$ can be accepted for surgery [1-3]. However, the incidence of neurological complications is increasing with the widespread use of bariatric surgery, especially in cases of acute axonal polyneuropathies [6].

AMSAN, a variant of GBS, is characterized by acute onset of distal weakness, loss of deep tendon reflexes, sensory symptoms, and is confirmed by ENMG [12-15].

Altered diet, reduced absorption, dysmotility, loss of gastric acid and intrinsic factors are important reasons in developing complications. Among them, 
B12 deficiency is the most common. A low B12 level has been reported at a ratio of $70 \%$ in these patients $[4,5]$. B12 levels were normal in our patients. In addition, deficiencies in B-group vitamins (niacin, pyridoxine and especially thiamine), vitamin E, copper, zinc, selenium, and folic acid are responsible for other nutritional factors in neurologic complications [6]. A deficiency in our evaluation was the lack of analysis of other B group vitamins and trace elements as this is not a part of our work routine. It has been reported that obese patients have preexisting micronutrient deficiencies and these patients' conditions deteriorate after surgery $[6,7]$. Also, a history of repeated attacks of vomiting have been reported as a risk factor for complications in bariatric cases [4]. Our patients had already taken vitamin B supplements and had no history of repeated attacks of vomiting after surgery.

Rapid and excessive weight loss may cause malnutrition related polyneuropathy [12-15]. In the first case, BMI decreased from $59.7 \mathrm{~kg} / \mathrm{m}^{2}$ to 40.3 $\mathrm{kg} / \mathrm{m}^{2}$ in six months. In the second case, it decreased from $50.5 \mathrm{~kg} / \mathrm{m}^{2}$ to $22.1 \mathrm{~kg} / \mathrm{m}^{2}$ in six months.

It is reported that neurological complications generally developed 3-20 months after bariatric surgery. This interval may be related to immunological disturbance caused by deficits of gastric incretins, and microelements deficiencies [11-14]. In our patients, AMSAN developed 3-4 months after surgery.

Diaz et al. [10] showed inflammatory changes in nerve biopsy in two cases with acute polyneuropathy after bariatric surgery. They also reported good response to IVIg in these cases. Also, Chang et al. [11] reported one patient who after being treated with plasmapheresis no response to IVIg followed. IVIg is a gold standard treatment of autoimmune neuropathies including GBS, chronic inflammatory demyelinating neuropathy and multifocal motor neuropathy. In addition, plasmapheresis may require a second line therapy in cases which are not responding to IVIg [9]. In light of these data, we preferred IVIg as the first line treatment. However, in the second case, plasmapheresis was needed because of no response to IVIg.

Currently, there is confusion in the naming of cases with post-bariatric polyneuropathies, using terms such as nutritional polyneuropathy, acute/subacute axonal polyneuropathy, and variants /mimics of GBS [12-14]. Using the term 'GBS variants related to malnutrition' could be more suitable in order to have a generally accepted term for these cases. Nutritional support before and after bariatric surgery, and routine biochemical evaluation at frequent intervals are very important to prevent these complications [7-10].

\section{CONCLUSION}

Our cases showed that IVIg should be kept in mind as a reliable treatment option for GBS variants caused by bariatric surgery.

\section{Informed consent}

Written informed consent was obtained from the patient for publication of this case report and any accompanying images.

\section{Conflict of interest}

The authors declared that there are no potential conflicts of interest with respect to the research, authorship, and/or publication of this article.

\section{REFERENCES}

[1] Buchwald H, Oien DM. Metabolic/bariatric surgery worldwide 2008. Obes Surg 2009;19:1605-11.

[2] Centre for Public Health Excellence at NICE (UK), National Collaborating Centre for Primary Care (UK): Obesity: The Prevention, Identification, Assessment and Management of Overweight and Obesity in Adults and Children. London 2006.

[3] NIH Consensus Development Conference Panel: Gastrointestinal surgery for severe obesity. Ann Intern Med 1991;115:956-61.

[4] Lawton AW, Frisard NE. Visual loss, retinal hemorrhages, and optic disc edema resulting from thiamine deficiency following bariatric surgery complicated by prolonged vomiting. Ochsner J 2017; $17: 112-4$

[5] Goodman JC. Neurological complications of bariatric surgery. Curr Neurol Neurosci Rep 2015;15:79.

[6] Papamargaritis D, Aasheim ET, Sampson B, le Roux CW. An overview of complications affecting the copper, selenium and zinc levels after bariatric surgery in patients recommended to take multivitamin-mineral supplementation. J Trace Elem Med Biol 
2015;31:167-72.

[7] Kazemi A, Frazier T, Cave M. Micronutrient related neurologic complications following bariatric surgery. Curr Gastroenterol Rep 2010;12:4:288-95.

[8] Punchai S, Hanipah ZN, Meister KM, Schauer PR, Brethauer SA, Aminian A. Neurologic nmnifestations of vitamin B deficiency after bariatric surgery. Obes Surg 2017;27:2079-82.

[9] Nobile-Orazio E, Terenghi F. IVIg in idiopathic autoimmune neuropathies: analysis in the light of the latest results. J Neurol 2005;252 Suppl 1:I7-13.

[10] Dias JC, Vidal CM, Freitas MRG. Inflammatory polyneuropathy after bariatric surgery: report of two cases. J Neurol Disord 2016;4:278.

[11] Chang CG, Adams-Huet B, Provost DA. Acute post-gastric reduction surgery (APGARS) neuropathy. Obes Surg 2004;14:182-9.
[12] Yasawy ZM, Hassan A. Post bariatric surgery acute axonal polyneuropathy: doing your best is not always enough. Ann Indian Acad Neurol 2017;20:309-12.

[13] Ishaque N, Khealani BA, Shariff AH, Wasay M. GuillainBarré syndrome (demyelinating) six weeks after bariatric surgery: a case report and literature review. Obes Res Clin Pract 2015;9:416-9.

[14] Landais AF. Rare neurologic complication of bariatric surgery: acute motor axonal neuropathy (AMAN), a severe motor axonal form of the Guillain Barré syndrome. Surg Obes Relat Dis $2014 ; 10: \mathrm{e} 85-7$.

[15] Kailasam VK, DeCastro C, Macaluso C, Kleiman A. Postbariatric surgery neuropathic pain (PBSNP): case report, literature review, and treatment options. Pain Med 2015;16:37482. 